\title{
Low-cost in vitro Screening Method for Digestibility of Pet Chews
}

\author{
${ }^{1}$ Timothy J. Bowser, ${ }^{2}$ Charles I. Abramson and ${ }^{3}$ Dwayne Bennett \\ ${ }^{1}$ Department of Biosystems and Agricultural Engineering, 110 FAPC, Oklahoma State University, \\ Stillwater, Oklahoma, 74078, and PetSci, LLC, Stillwater, Oklahoma, 74075 \\ ${ }^{2}$ Laboratory of Comparative Psychology and Behavioral Biology, Departments of Psychology and \\ Zoology, Oklahoma State University Stillwater, Oklahoma, 74078, \\ and PetSci, LLC, Stillwater, Oklahoma, 74075, and \\ ${ }^{3}$ Natural Polymer International Corporation (The Makers of N-Bone $\left.{ }^{\circledR}\right)$, Plano, Texas, 75074
}

\begin{abstract}
Four pet chew treats from four different manufacturers were tested for digestibility using in vitro tests in artificial gastric (stomach) and intestinal juices. In vitro tests were selected to determine the digestibility of dog chews for humane reasons and were conducted under conditions that were designed to simulate the digestive system of dogs. The purpose of the tests was to rapidly and inexpensively determines the rate of degradation of a dog chew in the canine digestive tract to assess product safety for dogs. Dog chew pet treats that are swallowed whole, or in part, should degrade rapidly in the canine digestive system to prevent potentially dangerous blockage. Tests were conducted on one $\mathrm{cm}$ and one-half $\mathrm{cm}$ cubes of dog chew products. Results of the tests clearly indicated that one product outperformed all others in short-term (under $4 \mathrm{hrs)} \mathrm{digestive} \mathrm{tests.} \mathrm{The} \mathrm{Bone-A-Mint} \mathrm{Wheat}$ Free formula degraded more rapidly than all other products in tests simulating canine gastric and intestinal juices. Tests conducted in this study will form the basis of an in vitro procedure that can be used as an industry standard to asses the safety of pet chew treats.
\end{abstract}

Key words: Chew, canine, digestibility, pet, in vitro, screening

\section{INTRODUCTION}

This report describes a simple, inexpensive and relatively quick in vitro test to help evaluate the digestibility of pet products. We evaluated this method by testing dog chew products. An artificial "stomach" and "intestine" that contained gastric and intestinal juices, respectively, was created. The product was placed in the artificial stomach and intestine and various dependent variables such as rate of digestion were calculated. The inspiration for the test came from Pavlov's conditioning experiments with dogs in which gastric secretions were redirected into a test tube ${ }^{[1]}$. Validation of in vitro test methods used to simulate digestion in animals has been established by many researchers ${ }^{[2-5]}$.

The test is designed as an alternative to using animals to evaluate the digestibility of pet chews and can easily be adapted to other animal models such as felines. Canine pet chews have attracted much interest resulting from recent media reports about pet illnesses and death ${ }^{[6-8]}$. Mortalities and illness were caused when chews that were swallowed whole or in large pieces lodged in the digestive tract of the animal remaining there for sustained time periods.

\section{MATERIALS AND METHODS}

Four commercially available dog chews were selected for this study and purchased from a local grocery store: Bone-A-Mint Wheat Free (Natural Polymer International Co.; The Makers of N-Bone ${ }^{\circledR}$, Plano, TX); Greenies ${ }^{\circledR}, S \&$ M NuTec, LLC (North Kansas City, MO); Waggin' Train ${ }^{\circledR}$ Freshies $^{\mathrm{TM}}$, ADI Pet, Inc. (Rancho, CA); and Nutri Dent ${ }^{\mathrm{TM}}$ Nylabone ${ }^{\circledR}$, Nylabone Products (Neptune, NJ). All products purchased were marked for medium-sized dogs, fresh (within code date if marked), with excellent appearance and in sound retail packaging. Products were tested in two sample sizes: 1.0 and $0.5 \mathrm{~cm}$ cubes. The $1.0 \mathrm{~cm}$ cube represented chunks that might accidentally be ingested by a large dog; the $0.5 \mathrm{~cm}$ cubes represented chunks that might accidentally be ingested by a smaller dog.

Procedure for in vitro degradation tests: A band saw (Model SM 400, Delta Machinery, TN) with a finetoothed blade (5.9 teeth per $\mathrm{cm}$ ) was used to cut the dog chews into 1.0 or $0.5 \mathrm{~cm}$ sample cubes. Cubes were cut from the entire length of the dog chew to provide sample representation from all portions of the product. Final dimensions of cubes were checked with a caliper (No. 144, General Hardware Mfg. Col, NY) and were

Corresponding Author: $\quad$ Dr. Timothy J. Bowser, Department of Biosystems and Agricultural Engineering, 110 FAPC, Oklahoma State University, Stillwater, OK 74078; Tel: 405-744-6688, Fax: 405-744-6313 


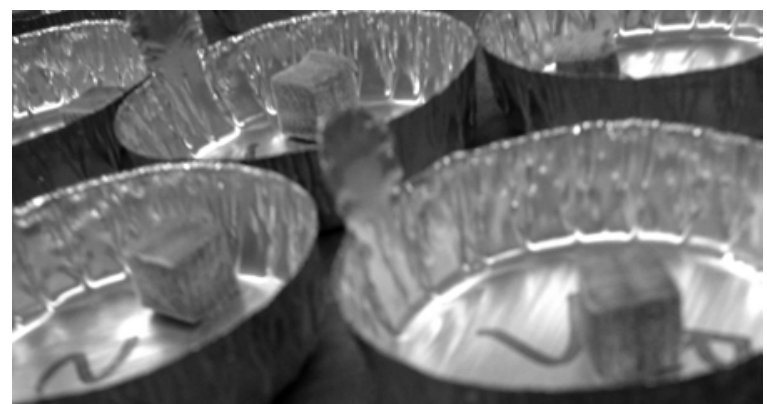

Fig. 1: Photograph of cubes cut from dog chews and placed in sample trays

within $+/-0.5 \mathrm{~mm}$. Figure 1 shows cut cubes in marked sample trays.

Artificial gastric or intestinal juice was prepared according to Okamoto et $a l_{.}^{[2]}$ and equilibrated at $38.6^{\circ} \mathrm{C}$, the internal body temperature of a dog. A $4 \times 2$ x 5 x 2 factorial designed was used with the 4 different chew products, 2 sizes $(1.0 \mathrm{~cm}, 0.5 \mathrm{~cm}$ cubes), 5 time periods (1, 2, 4, 8, $12 \mathrm{hrs)}$ and 2 types of juices (gastric or intestinal). An individual sample was placed in 250 $\mathrm{mL}$ of either gastric or intestinal juice and placed in an incubator or temperature-controlled environment of $38.6^{\circ} \mathrm{C}$ for $1,2,4,8$, or $12 \mathrm{hrs}$. Treatments were applied to samples in triplicate. Each hour all individual samples were agitated manually by shaking the container vertically 10 times.

After exposure to the required number of hours, the sample was drained. If a sample cube was observed to be intact, the contents of the container were drained through a number 20 mesh strainer and the cube gently rinsed with purified water. The cube was then placed on a drying tray. If particles were present (i.e., cube was not intact), the contents of the container were drained through coarse filter paper (P8, Fisher Scientific, Fairlawn, NJ) and the residual rinsed with purified water. The largest particles were removed from the filter paper and place on a drying tray. If two or more particles appeared to be of equal mass, they were placed on the same drying tray to later determine the particle with the greatest mass.

All drained samples were placed in a dryer at 38.6 ${ }^{\circ} \mathrm{C}$ and left to dry for $48 \mathrm{hrs}$ or until weight change over time was negligible. The final mass of the sample was taken as the mass of the heaviest remaining dried particle for each sample. After a determination of the final mass of the sample, we calculated the percent degradation of each sample according to the formula:

$\mathrm{PD}=(1-\mathrm{Fm} / \mathrm{Im}) * 100$

Where:

$\mathrm{Fm}=$ final mass, $\mathrm{g}$

$\mathrm{Im}=$ initial mass, $\mathrm{g}$

$\mathrm{PD}=$ percent degradation

\section{RESULTS AND DISCUSSION}

Figure 2 shows the average percent degradation for $1.0 \mathrm{~cm}$ dog chew cubes (all products tested) in artificial canine gastric juice over time. Figure 2 was plotted

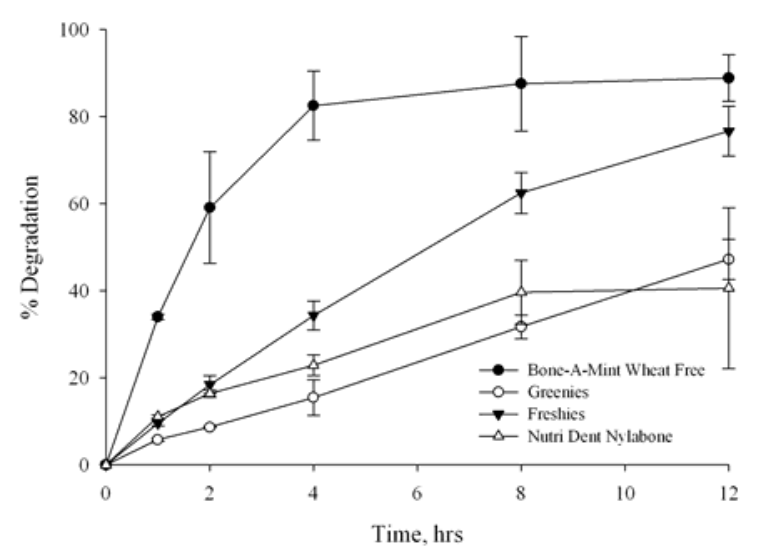

Fig. 2: Degradation of $1.0 \mathrm{~cm}$ dog chew cubes in artificial gastric juice

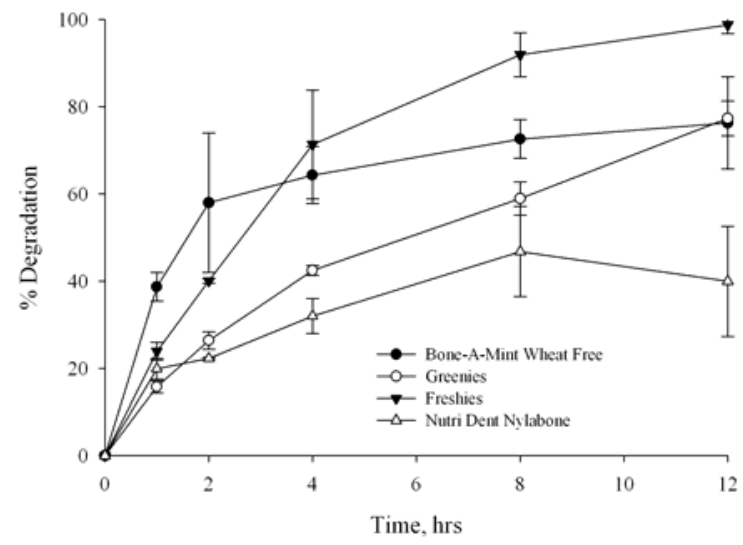

Fig. 3: Degradation of $0.5 \mathrm{~cm}$ dog chew cubes in artificial gastric juice

using SigmaPlot software (SigmaPlot for Windows version 10.0, Systat Software, Inc., San Jose, CA); the software calculates the mean of the three samples and error bars one standard deviation above and below the mean. Percent degradation was calculated using equation (1). Figure 3 is a similar graph for $0.5 \mathrm{~cm}$ dog chew cubes. Figure 4 shows the average percent degradation for $1.0 \mathrm{~cm}$ dog chew cubes (all products tested) in artificial canine intestinal juice over time with error bars of one standard deviation above and below the mean. Figure 5 is a similar graph for $0.5 \mathrm{~cm}$ dog chew cubes.

In vitro tests were designed to create a simple, accurate, inexpensive and repeatable method that could be used to test the digestibility of pet chews without harming animals. We believe that this goal was accomplished. Figure 2 indicated that the Bone-A-Mint Wheat Free dog chews outperformed all of the other products by degrading more rapidly in the artificial gastric juice. We believe this was a result of the unique wheat-free formulation and the absence of strong wheat gluten bonds present in the other products. Figure 3 revealed degradation curves that were similar in shape to those of Fig. 2, with the Bone-A-Mint Wheat Free 


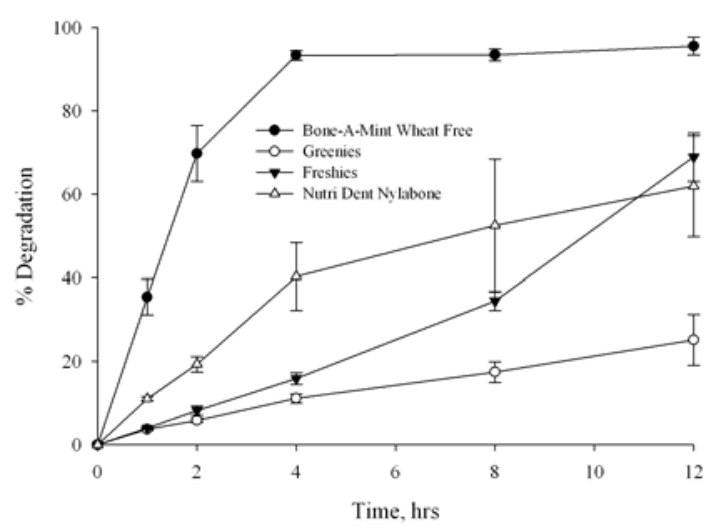

Fig. 4: Degradation of $1.0 \mathrm{~cm}$ dog chew cubes in artificial intestinal juice

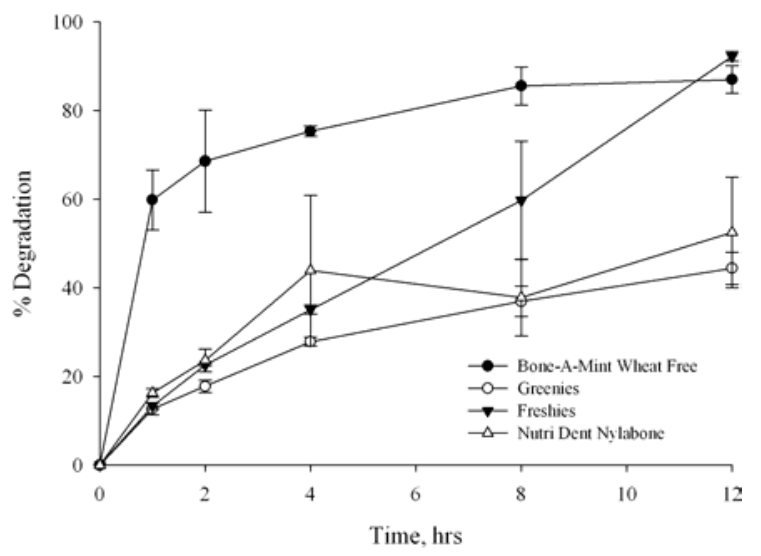

Fig. 5: Degradation of $0.5 \mathrm{~cm}$ dog chew cubes in artificial intestinal juice

product degrading the most rapidly within the first $4 \mathrm{hrs}$ of the test. Figures 4 and 5 revealed similar results for dog chew cubes in artificial intestinal juice.

The following discussion points relate to the test procedure and its simplification and future application and are based on information gained from laboratory experience with the scope of products tested:

Tests can be shortened to four to $6 \mathrm{hrs}$ based on the predictable degradation curve of each product and the realistic need for a rapid, convenient test and pet chew products that degrade rapidly after ingestion.

Degradation, rather than dissolution, is the metric of choice. During the experiments, it was observed that all of the products dissolved to differing degrees in the artificial gastric and intestinal juices, while some also disintegrated into smaller particles. Degradation takes into account both mechanisms of dissolution and disintegration.

Coarse filter paper was used to capture fine particles that disintegrated from the product sample cubes by straining the sample and digestive liquid through the filter paper. The filter paper and collected residue were dried in an oven, weighed and the data was recorded and used to determine the amount of the product that was dissolved into the liquid. This procedure was time consuming and tedious and was not useful in calculating overall product degradation. It is recommended to eliminate the use of filter paper in future tests. In lieu of filter paper, a \#20 mesh screen, or similar, can be used to strain product samples from digestive liquids.

It was noted that some of the samples of dog chew products softened appreciably when soaked in the digestive juices for the given time periods. Bone-AMint Wheat Free softened rapidly and began to disintegrate. Waggin' Train Freshies separated into distinct layers and became swollen. Products that soften may pass through the animal digestive tracts more readily than those that don't soften. A measurement of product softness may be a useful metric for future tests. A common texture measurement device (textureometer) could be used to measure the structural properties of samples after soaking in artificial digestive fluids.

\section{ACKNOWLEDGEMENTS}

This project was funded by Natural Polymer International Corporation (NPIC) which employs coauthor Bennett. Authors Abramson and Bowser are co-owners of PetSci, LLC, an independent company involved in the humane testing and evaluation of animal and pet products. All experiments were carried out by Bowser in PetSci's facilities.

\section{REFERENCES}

1. Pavlov, I.P., 1927. Conditioned Reflexes. Oxford, Oxford University Press.

2. Okamoto, Y., M. Nose, K. Miyatake, J. Sekine, R. Oura, Y. Shigemasa and S. Minami, 2001. Physical changes of chitin and chitosan in canine gastrointestinal tract. Carbohydrate Polymers, 44: 211-215.

3. Kalantzi, L., B. Polentarutti, T. Albery, D. Laitmer, B. Abrahamsson, J. Dressman and C. Reppas, 2005. The delayed dissolution of paracetamol products in the canine fed stomach can be predicted in vitro but it does not affect the onset of plasma levels. Int. J. Pharm., 269: 87-93.

4. Kho Kholeif, H., J. Larach, G. Thomforde, R. Dozois and J.R. Malagelada, 1983. A canine model for the study of gastric secretion and emptying after a meal. Dig. Dis. Sci., 28: 633-640.

5. Smeets, M.T. Watson, M. Minekus and R. Havenaar, 1998. A review of the physiology of the canine digestive tract related to the development of in vitro systems. Nutr. Res. Rev., 11: 45-69. 
6. Thompson, C., 2001. Chew toys unsafe for dogs? KOMO 1000 News Radio. Available: http://www.komotv.com/news/story.asp?ID=15561 . Accessed Sept. 20, 2006.

7. Havrelly, W., 2005. FDA investigates greenies following KIRO 7 investigation. KIRO 4 News. Available: http://www.kirotv.com/consumer/ 5436588/detail.html. Accessed Sept. 20, 2006.
8. Hunter, G. and P. Malbran. 2006. Owners: dog treats killed our pets. CNN News. Available: http://www.cnn.com/2006/US/02/14/dangerous.do gtreat/index.html. Accessed Sept. 20, 2006. 